\title{
IL-10 plays a central regulatory role in the cytokines induced by hepatitis $C$ virus core protein and polyinosinic acid:polycytodylic acid
}

\author{
Xiaoli Pang a,b ${ }^{\text {, }}$ Zhaoxia Wang ${ }^{b}$, Naicui Zhai ${ }^{c}$, Qianqian Zhang ${ }^{\mathrm{a}}$, Hongxiao Song ${ }^{\mathrm{c}}$, Yujiao Zhang ${ }^{\mathrm{a}}$, Tianyang Li ${ }^{\mathrm{c}}$, \\ Haijun $\mathrm{Li}^{\mathrm{c}}$, Lishan $\mathrm{Su}^{\mathrm{d}, \mathrm{e}}$, Junqi $\mathrm{Niu}^{\mathrm{a}, *}$, Zhengkun $\mathrm{Tu}^{\mathrm{c}, *}$ \\ a Department of Hepatology, The First Hospital of Jilin University, Changchun, Jilin 130021, China \\ b Department of Pediatric Gastroenterology, The First Hospital of Jilin University, Changchun, Jilin 130021, China \\ c Translational Medicine Research Institute, The First Hospital of Jilin University, Changchun, Jilin 130021, China \\ d Lineberger Comprehensive Cancer Center, The University of North Carolina at Chapel Hill, Chapel Hill, NC, United States \\ e Department of Microbiology and Immunology, The University of North Carolina at Chapel Hill, Chapel Hill, NC, United States
}

\section{A R T I C L E I N F O}

\section{Article history:}

Received 9 February 2016

Received in revised form 15 June 2016

Accepted 17 June 2016

Available online 21 June 2016

\section{Keywords:}

IL-10

Inflammatory cytokines

Antiviral cytokines

A central role

Negative feedback

\begin{abstract}
A B S T R A C T
Hepatitis C virus (HCV) can cause persistent infection and chronic liver disease, and viral factors are involved in $\mathrm{HCV}$ persistence. $\mathrm{HCV}$ core protein, a highly conserved viral protein, not only elicits an immunoresponse, but it also regulates it. In addition, HCV core protein interacts with toll-like receptors (TLRs) on monocytes, inducing them to produce cytokines. Polyinosinic acid:polycytodylic acid (polyI:C) is a synthetic analogue of doublestranded RNA that binds to TLR3 and can induce secretion of type I IFN from monocytes. Cytokine response against $\mathrm{HCV}$ is likely to affect the natural course of infection as well as HCV persistence. However, possible effects of cytokines induced by HCV core protein and polyI: $C$ remain to be investigated. In this study, we isolated CD14 ${ }^{+}$ monocytes from healthy donors, cultured them in the presence of HCV core protein and/or polyl:C, and characterized the induced cytokines, phenotypes and mechanisms. We demonstrated that HCV core protein- and polyI:C-stimulated CD14 ${ }^{+}$monocytes secreted tumor necrosis factor- $\alpha$ (TNF- $\alpha$ ), interleukin (IL)-1 $1 \beta$, IL- 10 , and type I interferon (IFN). Importantly, TNF- $\alpha$ and IL-1 $\beta$ regulated the secretion of IL-10, which then influenced the expression of signal transducer and activator of transcription 1 (STAT1) and interferon regulatory factor 1 (IRF1) and subsequently the production of type I IFN. Interestingly, type I IFN also regulated the production of IL-10, which in turn inhibited the nuclear factor (NF)-KB subunit, reducing TNF- $\alpha$ and IL-1 $\beta$ levels. Therefore, IL-10 appears to play a central role in regulating the production of cytokines induced by HCV core protein and polyl:C.
\end{abstract}

\section{Introduction}

Hepatitis $\mathrm{C}$ virus (HCV) infection in the majority of affected individuals becomes chronic, and the mechanisms responsible for persistent $\mathrm{HCV}$ infection are not completely understood. It has been suggested that the host's insufficient immune response and particularly weak cellular immunity lead to viral persistence [1,2]. However, viral factors are also involved in HCV pathogenesis.

Accumulating evidence suggests that HCV proteins can suppress the host's immune responses by interfering with immune cell functions [35]. The core protein is a highly conserved structural protein that is required to assemble the viral nucleocapsid, which encapsulates the viral RNA genome. HCV core protein also interacts with HCV envelope proteins to initiate virus particle assembly [6,7]. In addition, it regulates cellular transcription, transformation, signal transduction, and innate

\footnotetext{
* Corresponding authors.

E-mail addresses: junqiniu2013@126.com (J. Niu), zhengkuntu2013@126.com (Z. Tu).
}

immunity. Moreover, HCV core protein is believed to engage in virusmediated pathogenesis through modulating apoptosis, cell growth, and reactive oxygen species production $[6,8]$. HCV core-dependent deregulation of the Janus kinase-signal transducer and activator of transcription (STAT) signaling pathway, which suppresses HCV-induced innate immunity, has been suggested [9-11].

Monocytes are important immune cells that defend the host against numerous pathogens as well as initiate and control adaptive immunity [12]. These cells express a panel of receptors including toll-like receptors (TLRs), which bind to diverse pathogen-derived products, thus triggering the immune response to a broad range of bacterial and viral pathogens $[13,14]$. HCV core protein and double-stranded RNA can interact with TLR2 and TLR3 on monocytes and induce large quantities of proinflammatory and anti-inflammatory cytokines, which may lead to hepatic inflammation that can facilitate HCV infection clearance.

Peripheral monocytes produce interleukin (IL)-10, which can inhibit the HCV immune response $[15,16]$. The IL-10 level has been associated with an elevated serum alanine transaminase and/or histological 
grade in HCV patients [17]. In addition, it has been correlated with outcomes of chronic viral infection [18]. One study has revealed that type I interferon (IFN) can induce IL-10 production and the subsequent STAT3 phosphorylation, thus further inhibiting the expression of proinflammatory cytokines including IL-1 $\beta$, tumor necrosis factor- $\alpha$ (TNF- $\alpha$ ), and IL-12 [19]. It is unclear whether proinflammatory cytokines regulate the IL-10 level and functions or further modulate type I IFN expression. In this study, we investigated the responses of monocytes to HCV core protein and polyinosinic acid:polycytodylic acid (polyl:C) which could simulate HCV RNA. And we showed that proinflammatory cytokines induce the upregulation of IL-10 and the subsequent inhibition of type I IFN production, in turn, type I IFN increase the secretion of IL10 and then inhibits proinflammatory cytokines production. IL-10 appears to play a central role in the regulatory network of inflammatory and antiviral cytokines.

\section{Materials and methods}

\subsection{Reagents}

We purchased the experimental reagents from the following sources: HCV Core Antigen [amino acids 2-192] Recombinant and $\beta$ galactosidase (Meridian Life Science, Inc., Saco, ME, USA); polyI:C (Invivogen, San Diego, CA, USA); anti-human IL-10 Functional Grade Purified, Anti-Human TNF alpha Functional Grade Purified, AntiHuman IL-1 beta Functional Grade Purified, Anti-Human IFN beta Functional Grade Purified, and Anti-Human IFN- $\alpha$ (eBioscience, San Diego, CA, USA); human IL-10 ELISA ready-SET-Go, human TNF alpha ELISA ready-SET-Go, human IL-1 beta ELISA ready-SET-Go (eBioscience, San Diego, CA, USA); VeriKine TM Human Interferon beta/alpha ELISA Kit (PBL Assay Science, Piscataway, NJ, USA); CD14 microbeads (Miltenyi Biotec, Inc., Auburn, CA, USA); PE/Cy7-conjugated anti-human HLADR, PerCP-conjugated anti-human HLA-DR, PE-conjugated anti-human CD38, PE/Cy7-conjugated anti-human PD-L1 (BD Biosciences, Franklin Lakes, NJ, USA); Human Fc Receptor Binding Inhibitor Purified (eBioscience, San Diego, CA, USA); Easypure RNA kit (Transgen Biotech, Beijing, China); Faststart Universal SYBR Green Master (ROX) (Roche, Basel, Switzerland); and TransScript All-in-One First-Strand cDNA Synthesis SuperMix for qPCR (Transgen Biotech, Beijing, China).

\subsection{Isolation of monocytes from peripheral blood}

Peripheral blood concentrated lymphocytes of Healthy blood donors were provided by the Changchun Blood Center, and informed consent was obtained according to the protocols of the Changchun Blood Center. Peripheral blood mononuclear cells (PBMCs) were isolated and purified by density gradient centrifugation using lymphocyte separation medium.

\subsection{Purity and culture of $C D 14^{+}$monocytes}

The $\mathrm{CD} 14^{+}$cells were isolated and purified using CD14 microbeads. The purities of the $\mathrm{CD} 14^{+}$cells were determined by flow cytometry, and the purity was always $>90 \%$. A half million purified $\mathrm{CD} 14^{+}$monocytes were cultured in 96-well flat-bottomed plates with complete RPMI 1640 containing $10 \%$ fetal calf serum and $100 \mathrm{IU} / \mathrm{mL}$ penicillin and streptomycin at $37{ }^{\circ} \mathrm{C}$ with $5 \% \mathrm{CO}_{2}$.

\subsection{Flow cytometry}

Cell staining and analyses were performed as described previously $[20,21]$. In brief, CD14 ${ }^{+}$monocytes were cultured for 2 days in the presence of HCV core protein (final concentration of $10 \mu \mathrm{g} / \mathrm{mL}$ ) or polyI:C (final concentration of $50 \mu \mathrm{g} / \mathrm{mL}$ ) and then resuspended in staining buffer (phosphate-buffered saline containing $0.5 \%$ bovine serum albumin) and preincubated with FcR blocking reagent for 15 min at $4{ }^{\circ} \mathrm{C}$.
Next, the cells were stained with PE/Cy7-conjugated HLA-DR or PerCP-conjugated anti-human HLA-DR, PE-conjugated anti-human CD38, or PE/Cy7-conjugated anti-human PD-L1 and incubated for 20$40 \mathrm{~min}$. The cells were then washed with staining buffer and resuspended in phosphate-buffered saline containing $1 \%$ paraformaldehyde and analyzed on a flow cytometer (BD LSRFortessa cell analyzer, USA). The acquired data were analyzed with Flowjo (Treestar software).

\subsection{Analysis of the cytokines}

The cytokine concentrations were measured using ELISA kits. One hundred microliters of supernatant was used for each analysis. All samples were assayed in duplicate.

\section{6. $q P C R$}

The $\mathrm{CD} 14^{+}$monocytes were cultured and treated with HCV core protein and polyl: $C$ or neutralizing antibodies (the final concentrations of anti-human IL-10 and IL- $1 \beta$ were $10 \mathrm{ng} / \mu \mathrm{L}$, anti-human TNF- $\alpha$ was used at $12.5 \mathrm{ng} / \mu \mathrm{L}$, and anti-human IFN- $\alpha$ and IFN- $\beta$ were used at $5 \mathrm{ng} / \mu \mathrm{L}$, according to the efficiency of neutralization by ELISA). The cells were then harvested using an Easypure RNA kit and reverse transcribed into complementary DNA (cDNA) per the manufacturer's instructions. The cDNAs were amplified by qPCR in a Real-Time PCR System (Applied Biosystems StepOnePlus ${ }^{\mathrm{TM}}$, Life Technologies, USA). Target gene expression was determined using the comparative cycle threshold $(\triangle \triangle \mathrm{CT})$ method and normalized to GAPDH. The primers are shown in Table 1 .

\subsection{Statistical analysis}

Statistical analyses were performed using SPSS 18.0. Differences were evaluated using one-way analysis of variance (ANOVA), followed by Bonferroni's multiple-comparison test. $\mathrm{p}<0.05$ was considered significant and is indicated by an asterisk $\left(^{*}\right)$.

\section{Results}

3.1. HCV core protein- and polyinosinic acid:polycytodylic acid (polyI:C)induced secretion of cytokines in $\mathrm{CD} 14^{+}$monocytes

Monocytes constitute $10-20 \%$ of the total PBMCs. Monocytes are characterized by their phenotypic CD14 expression, commonly called $\mathrm{CD} 14^{+}$monocytes.

In the present study, we first investigated the expression levels of TLRs on PBMCs and found that the PBMCs expressed TLR2, TLR3, and

Table 1

Primers used for qPCR analysis of gene expression.

\begin{tabular}{|c|c|c|}
\hline Gene & Primers & Product size \\
\hline \multirow[t]{2}{*}{ GAPDH } & PF: 5'-CCACCTTTGACGCTGGG-3' & 66 bp \\
\hline & PR: 5'-CATACCAGGAAATGAGCTTGACA-3' & \\
\hline \multirow[t]{2}{*}{ IL-10 } & PF: 5'-GGGAGAACCTGAAGACCCTCA-3' & $72 \mathrm{bp}$ \\
\hline & PR: 5'-TGCTCTTGTTTTCACAGGGAAG-3' & \\
\hline \multirow[t]{2}{*}{ TNF- $\alpha$} & PF: 5'-ATCCTGGGGGACCCAATGTA-3' & $112 \mathrm{bp}$ \\
\hline & PR: 5'-AAAAGAAGGCACAGAGGCCA-3' & \\
\hline \multirow[t]{2}{*}{ IL-1 $\beta$} & PF: 5'-CAGAAGTACCTGAGCTCGCC-3' & $153 \mathrm{bp}$ \\
\hline & PR: 5'-AGATTCGTAGCTGGATGCCG-3' & \\
\hline \multirow[t]{2}{*}{ IFN- $\beta$} & PF: 5'-TGGCTGGAATGAGACTATTGTTGAG-3' & 76 bp \\
\hline & PR: 5'-CAGGACTGTCTTCAGATGGTTTATCT-3' & \\
\hline \multirow[t]{2}{*}{ STAT1 } & PF: 5'-CGGCTGAATTTCGGCACCT-3' & $81 \mathrm{bp}$ \\
\hline & PR:5'-CAGTAACGATGAGAGGACCCT-3' & \\
\hline \multirow[t]{2}{*}{ STAT3 } & PF: 5'-ATTGCCCGGATTGTGGCCCG-3' & 117 bp \\
\hline & PR: 5'-CTCCGTCACCACGGCTGCTG-3' & \\
\hline \multirow[t]{2}{*}{ IRF1 } & PF: 5'-AAAAGGAGCCAGATCCCAAGA-3' & $128 \mathrm{bp}$ \\
\hline & PR: 5'-CATCCGGTACACTCGCACAG-3' & \\
\hline \multirow[t]{2}{*}{$N F \kappa B$} & PF: 5'-TGGGCTACACCGAAGCAAT-3' & 69 bp \\
\hline & PR: 5'-GGGCCTGAGAGGTGGTCTT-3' & \\
\hline
\end{tabular}


TLR4 (data not shown). Then CD14 ${ }^{+}$monocytes were separated and cultured alone, or with $\beta$-galactosidase ( $\beta$-gal as control) or with HCV core protein and/or polyI:C. Our previous study showed that the peak secretion of IL-10 occurred on the second day of incubation, but the peaks of TNF- $\alpha$, IL- $1 \beta$ and IFN- $\beta$ secretion occurred on the first day $[22]$. Thus, we detected the IL-10 level in the medium collected on the second day and the levels of TNF- $\alpha$, IL- $1 \beta$, and IFN- $\beta$ in the medium collected on the first day after the addition of HCV core protein or polyI:C. Our results showed that HCV core protein could induce the secretion of TNF- $\alpha$, IL-1 $\beta$, and IL-10, and polyI:C can induce secretion of type I IFN in $\mathrm{CD}_{14}{ }^{+}$monocytes. We also found that HCV core protein inhibited polyI:C-induced type-I IFN production. However, polyI:C acted synergistically with HCV core protein to induce secretion of IL-10 in the monocytes but inhibited HCV core protein-induced TNF- $\alpha$ and IL- $1 \beta$ secretion (Fig. 1).

3.2. $\mathrm{HCV}$ core protein and polyI:C affect the phenotypes of $\mathrm{CD} 14^{+}$ monocytes

To further characterize the phenotypes of monocytes induced by $\mathrm{HCV}$ core protein or polyI:C, flow cytometry was used to determine phenotypic expression, including human leukocyte antigen-D related (HLADR), cluster of differentiation 38 (CD38), and programmed death-ligand 1 (PD-L1), which reflect the host's immune response $[23,24]$ related to HCV persistence [25-32].

We found that the optimal concentrations of HCV core protein and polyl:C for induction were $10 \mu \mathrm{g} / \mathrm{mL}$ and $50 \mu \mathrm{g} / \mathrm{mL}$, respectively, and that the number of dead cells became fewer on day 2, resulting in better phenotypic expression. Our results also showed that HCV core protein inhibited HLA-DR expression but upregulated PD-L1 expression. PolyI:C induced the expression of CD38, HLA-DR, and PD-L1 in CD14 ${ }^{+}$monocytes. Meanwhile, HCV core protein inhibited polyI:C-induced CD38 and HLA-DR expression. However, HCV core protein synergized with
polyl:C to upregulate PD-L1 expression in monocytes. Therefore, HCV core protein and polyI: $C$ could modulate the monocyte phenotypes, which may alter the monocyte functions (Fig. 2).

\subsection{HCV core protein and polyI:C modulate the phenotypes of $C D 14^{+}$ monocytes through cytokines}

We investigate whether cytokines involved in the regulation on phenotypes of $\mathrm{CD} 14^{+}$monocytes.

Our results indicated HCV core protein- and polyl:C-induced cytokine secretion in $\mathrm{CD} 14^{+}$monocytes. Moreover, these cytokines appear to modulate the monocyte phenotype. Neutralizing antibodies were used to demonstrate the direct contribution of specific cytokines to cell surface marker expression. When IL-10 or TNF- $\alpha$ expression was blocked, HLA-DR expression increased; but when type I IFN was blocked, HLA-DR expression decreased. In addition, the expression of CD38 and PD-L1 was decreased when TNF- $\alpha$, IL-1 $1 \beta$, or type I IFN (Fig. 3) was blocked. Therefore, HCV core protein and polyI:C modulated the phenotypes of $\mathrm{CD} 14^{+}$monocytes via cytokines.

\subsection{IL-10 is required for the regulation of cytokines induced by HCV core protein and polyI:C}

Given that cytokines can modulate cell phenotypes, next we studied the interaction of IL-10 and other cytokines. Our results revealed that blocking of IL-10 resulted in increased levels of TNF- $\alpha$, IL-1 $\beta$, and IFN- $\beta$. On the other hand the IL-10 level was decreased when TNF- $\alpha$, IL-1 $\beta$, or type I IFN was blocked (Fig. $4 A$ ). That is, TNF- $\alpha$, IL-1 $\beta$, or type I IFN induced the production of IL-10, and in turn, IL-10 inhibited the secretion of TNF- $\alpha$, IL-1 $1 \beta$, and type I IFN. The quantitative detection of cytokine mRNAs was consistent with the detected protein levels (Fig. 4B).
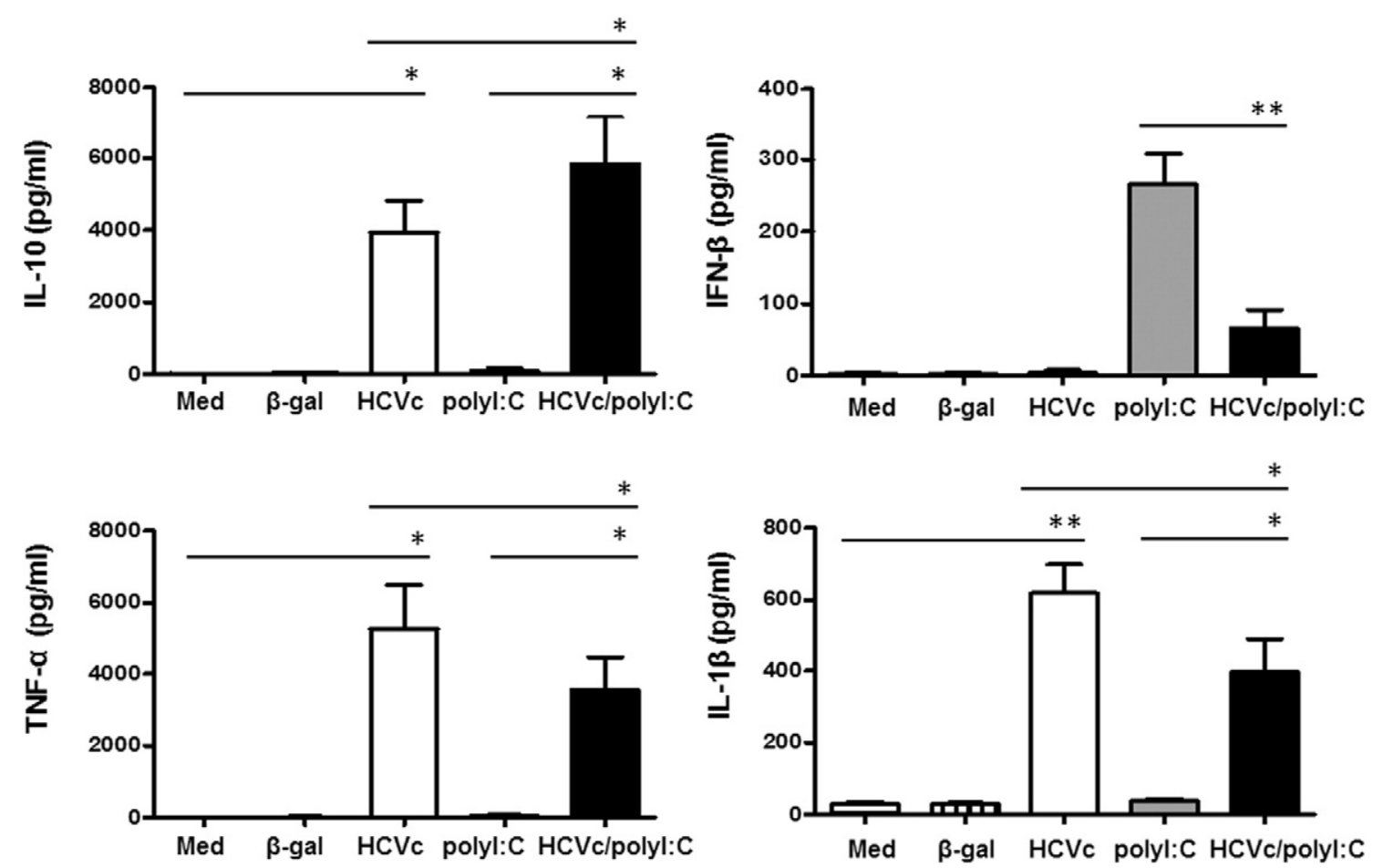

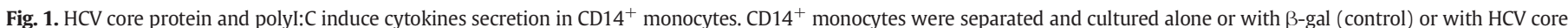

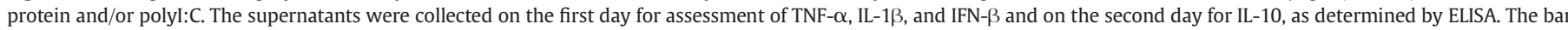

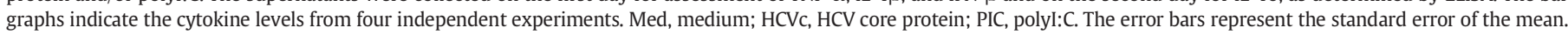
${ }^{*} \mathrm{p}<0.05,{ }^{* *} \mathrm{p}<0.01$. 

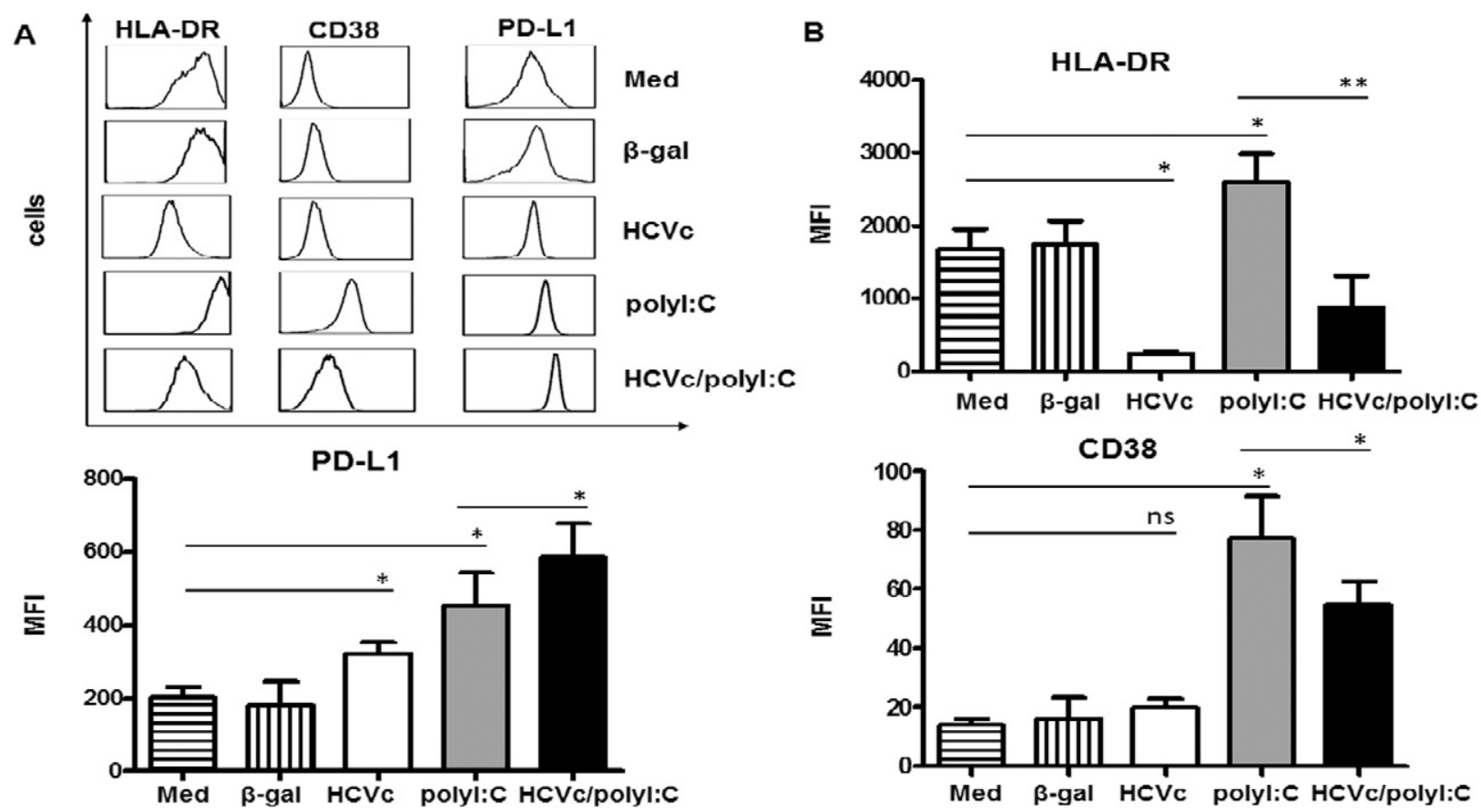

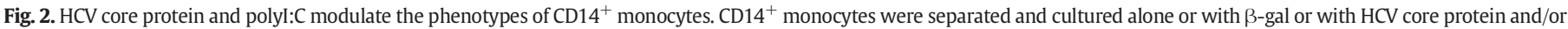

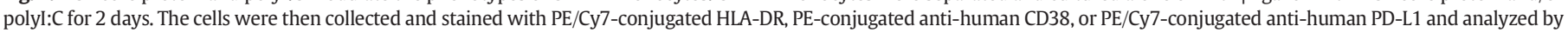

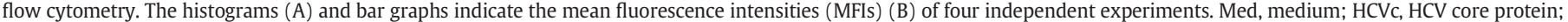
PIC:polyl:C. The error bars represent the standard error of the mean. ${ }^{*} \mathrm{p}<0.05,{ }^{* *} \mathrm{p}<0.01$, and ns indicates nonsignificant.

3.5. IL-10 regulates the secretion of TNF- $\alpha$ and IL-1 $\beta$ by inhibiting nuclear factor (NF)- $\kappa B$ and the production of type I IFN by inhibiting STAT1 and interferon regulatory factor 1 (IRF1)

We further studied the immunomodulatory mechanism of IL-10. Several candidate transcription factors that are likely involved in IL-10 production by macrophages have been described [33-36]. Our results revealed that the expression levels of NFKB, STAT1 and IRF1 were increased when IL-10 was blocked, as assessed by quantitative polymerase chain reaction ( $\mathrm{qPCR}$ ); however, no significant change was detected in the expression of STAT3 (Fig. 5).
In combination with other studies, our results led us to the conclusion that TNF- $\alpha$, IL-1 $\beta$, and type I IFN induced IL-10 secretion and that IL-10 subsequently inhibited type I IFN production by downregulating STAT1 and IRF1, thus inhibiting the secretions of TNF- $\alpha$ and IL-1 $\beta$ by inhibiting NFkB. These factors form a regulatory network centered on IL-10.

\section{Discussion}

In this study, we examined whether HCV core protein and polyI:C interact with TLRs on monocytes or modulate the biological functions
HLA-DR

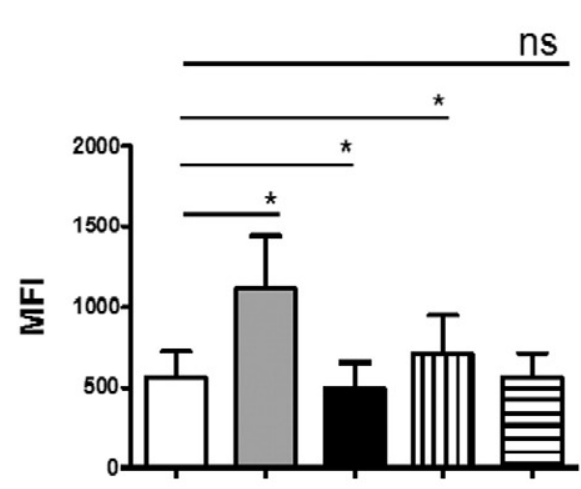

$\mathrm{W} / \mathrm{O}(\mathrm{HCV}+\mathrm{PIC})$
CD38

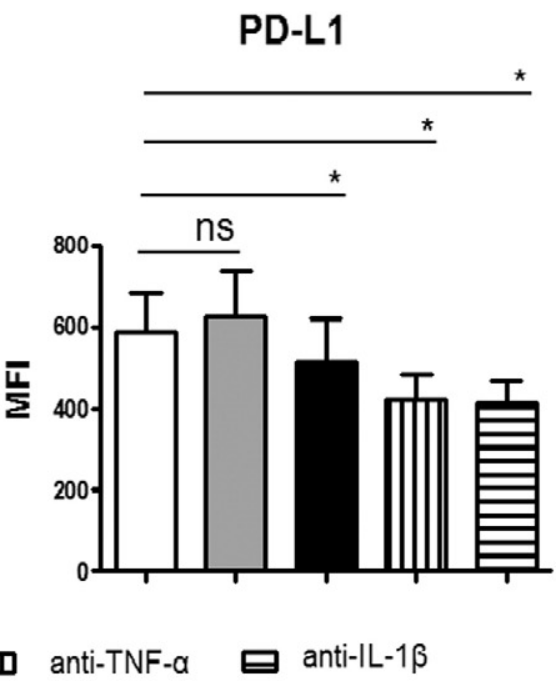

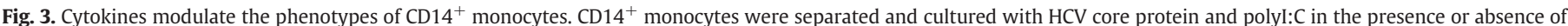

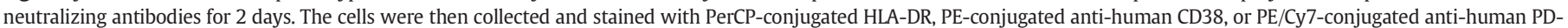

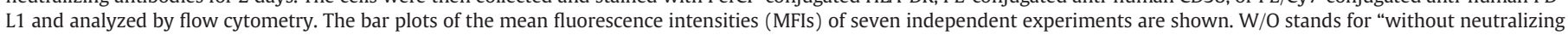
antibody" while all groups received both HCVc and PIC. The error bars represent the standard error of the mean. ${ }^{*} \mathrm{p}<0.05$, ${ }^{* *} \mathrm{p}<0.01$, ${ }^{* * *} \mathrm{p}<0.001$, and ns indicates nonsignificant. 
A

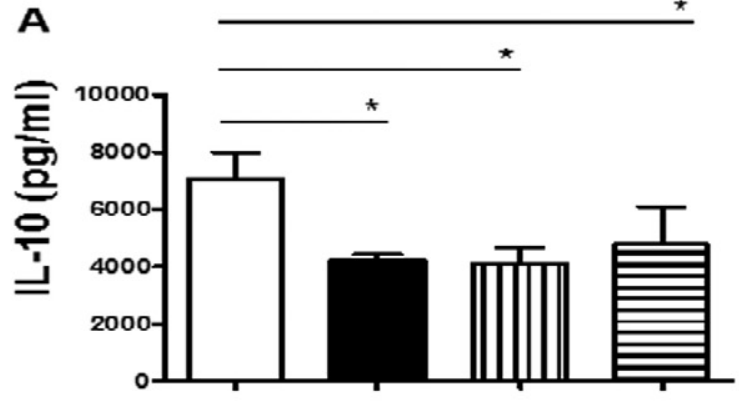

ns

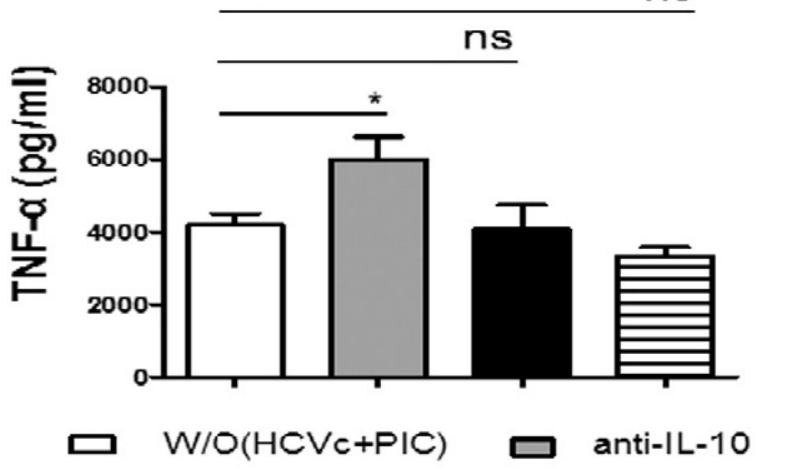

B

IL-10

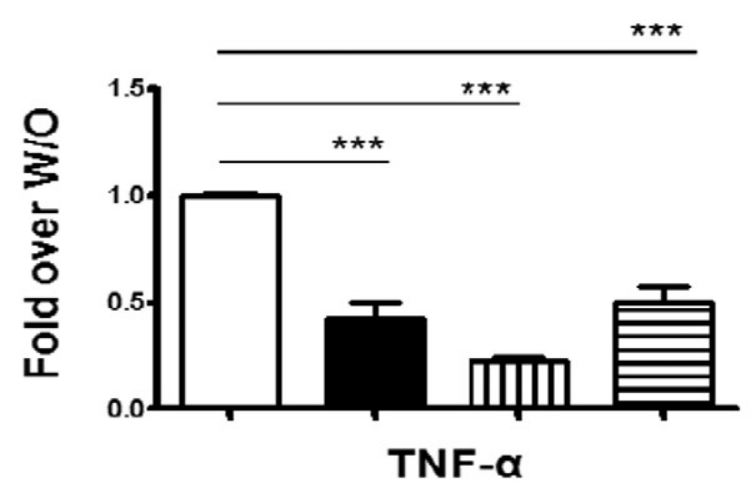

ns

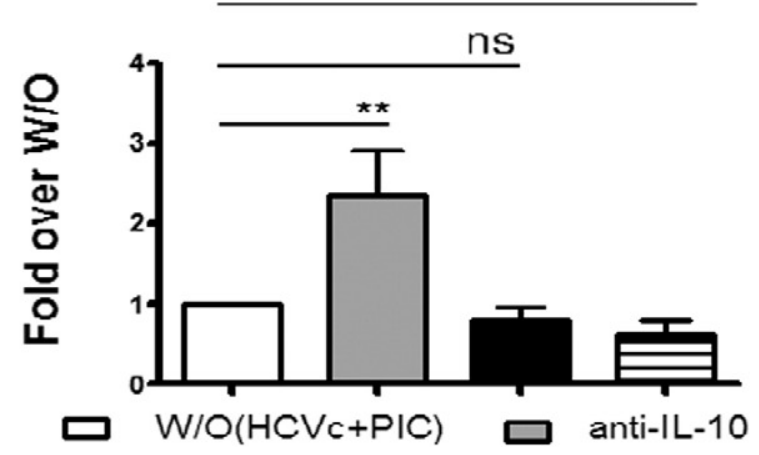

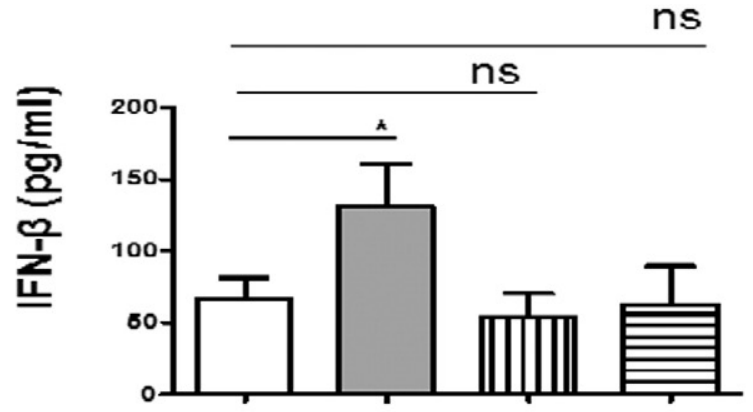

ns

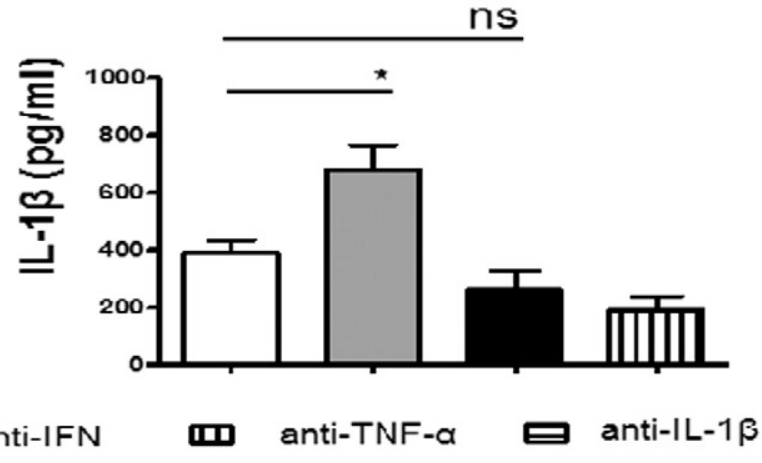

IFN- $\beta$ ns
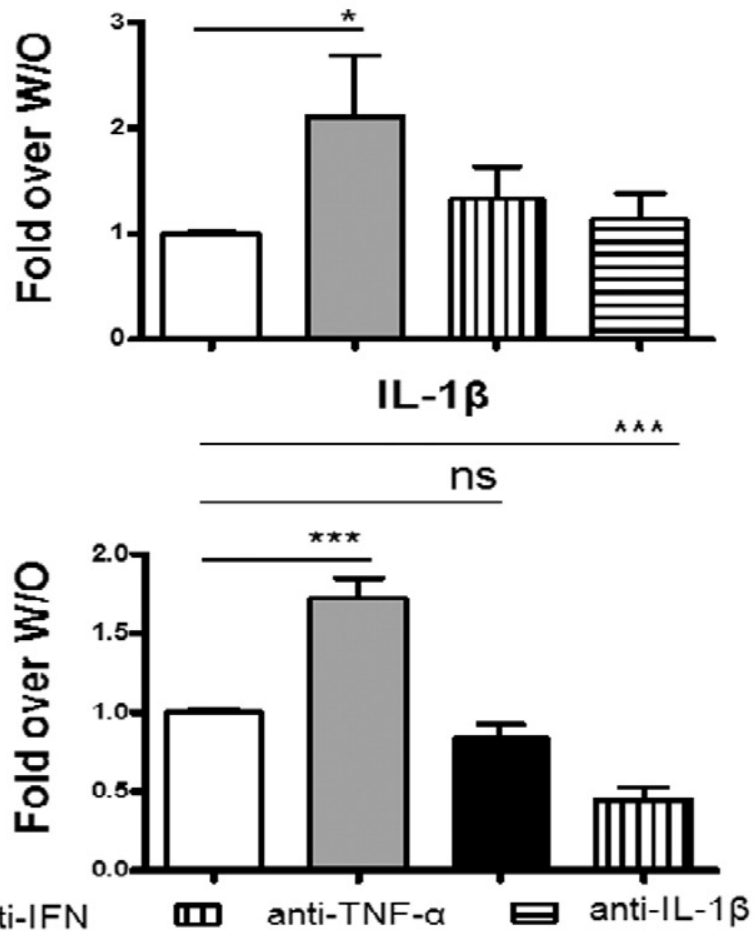

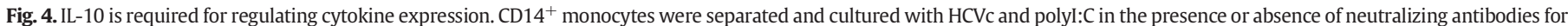

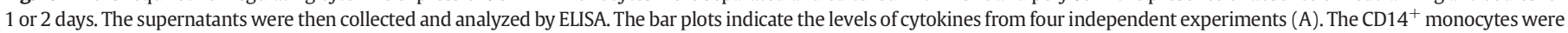

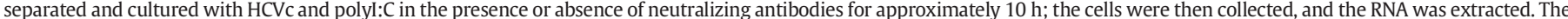

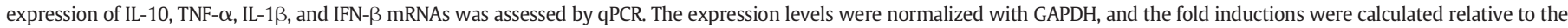

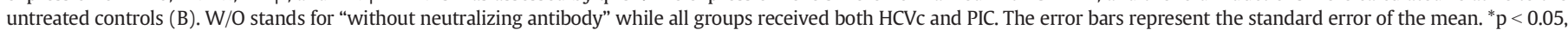
${ }^{* *} \mathrm{p}<0.01,{ }^{* * *} \mathrm{p}<0.001$, and ns indicates nonsignificant. 


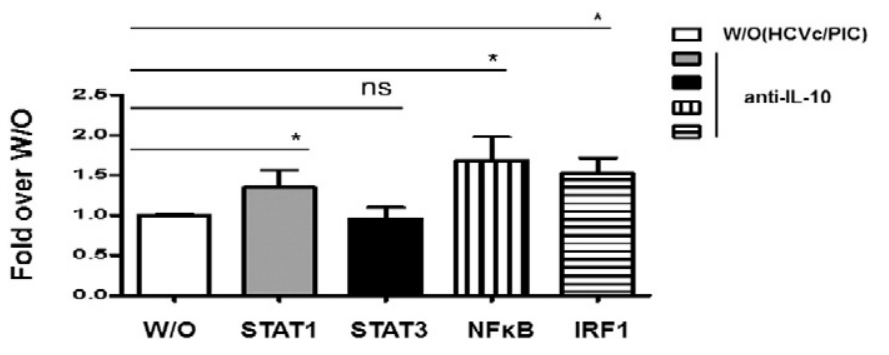

Fig. 5. IL-10 regulates cytokine secretion through negative feedback. CD14 ${ }^{+}$monocytes were separated and cultured with HCVc and polyI:C in the presence or absence of neutralizing antibodies for $10 \mathrm{~h}$; the cells were then collected, and the RNA was extracted. The expression of STAT1, STAT3, NFKB, and IRF1 mRNA was assessed by RTPCR. The expression levels were normalized with GAPDH, and the fold inductions were calculated relative to the untreated controls. W/O stands for "without neutralizing antibody" while all groups received both HCVc and PIC. The error bars represent the standard error of the mean. ${ }^{*} \mathrm{p}<0.05$, and ns indicates nonsignificant.

of monocytes that might directly or indirectly contribute to persistent HCV infection. Many studies showed that HCV core protein bound to TLR2 [37,38], while polyI:C could activate TLR3 [39]. We found that TLR2, TLR3, and TLR4 were expressed on purified CD14 ${ }^{+}$monocytes (data not shown). Our results also showed that HCV core protein induced the secretion of the inflammatory cytokines TNF- $\alpha$ and IL-1 $\beta$ as well as the immunomodulatory cytokine IL-10. Meanwhile, polyl:C induced type I IFN, which is currently used for treating chronic HCV infection.

Cytokines can regulate both phenotypes and functions of cells. IL-37 induces a phenotypic shift in human acute monocytic leukemia-derived macrophages toward a high-CD206 ${ }^{+}$and low-CD86 ${ }^{+}$macrophage subtype [40]. IL-15 strongly induces the expression of CD69 and CD54 in approximately $30 \%$ of natural killer cell subsets and downregulates CD62L expression, which is indicative of functional activation [41]. Monocytes that had been treated with IL-10 in vitro had significantly decreased HLA-DR expression, thus resulting in expansion of the CD14 ${ }^{+}$HLA$\mathrm{DR}^{\text {low/- }}$ population [42]. Our results revealed that TNF- $\alpha$, IL-1 $\beta$, IL-10 and IFN-Binduced by HCV core protein and polyI: $\mathrm{C}$ appeared to modulate the expression of HLA-DR, CD38 and PD-L1, and might alter their biological functions.

Most of important, IL-10 appeared to play a central role in the regulatory circuit of inflammatory and antiviral cytokines. we found that IL10 not only inhibited the production of TNF- $\alpha$ and IL- $1 \beta$ induced by $\mathrm{HCV}$ core protein but also reduced the secretion of type I IFN stimulated by polyI:C. IL-10 may limit hepatic inflammation and injury, but it also potentially slows down $\mathrm{HCV}$ infection clearance. Interestingly, we also found that TNF- $\alpha$, IL-1 $\beta$ and type I IFN elevated IL-10 expression, which in a negative feedback mechanism reduced the production of TNF- $\alpha$, IL-1 $\beta$, and type I IFN. Our findings are in agreement with published data that the production and signaling pathway of type I IFN are involved in lipopolysaccharide-induced IL-10 production that subsequently suppresses proinflammatory cytokine genes [19].

To gain insight into the mechanisms of IL-10 that regulates these cytokines, several candidate transcription factors have been described. One study reported that type I IFN induced the production of IL-10 and subsequent STAT3 phosphorylation in murine bone-marrow-derived macrophages and additionally inhibited the expression of proinflammatory genes [19]. Another study has found that IL-10 inhibits the expression of both IFN- $\alpha$ - and IFN- $\gamma$-induced genes by suppressing tyrosine phosphorylation of STAT1 [43]. We cultured CD14 ${ }^{+}$monocytes in the presence of $\mathrm{HCV}$ core protein and polyI:C. We found that NFkB, STAT1, and IRF1 were a few components that regulate IL-10. Our results suggest that IL-10 might inhibit NFkB, which leads to the suppression of TNF- $\alpha$ and IL-1 $\beta$ while downregulating STAT1 and IRF1, which reduces the type I IFN level. However, the expression of STAT3 was not affected. We believe that this difference was attributable to a species difference.
In conclusion, peripheral monocytes secreted TNF- $\alpha$, IL-1 $1 \beta$, and type I IFN upon induction with HCV core protein and polyI:C. All three cytokines increased IL-10 production, which in a negative feedback mechanism inhibited the expression of proinflammatory cytokines and type I IFN. Our results suggested that IL-10 countered hepatic inflammation and clearance of HCV infection and that it may represent a factor contributing to the persistence of HCV infection.

\section{Conflicts of interest}

The authors declare no conflicts of interest.

\section{Acknowledgments}

This work was supported in part by the National Science and Technology Major Project (2013ZX10002008, 2012ZX10002007), the National Basic Research Program of China (973 Program) (2015CB554304) and the National Natural Science Foundation of China (grant numbers 81373057, 81200289, 81301472, 81270484, 81202377, and 81301415). We would like to thank Medjaden Bioscience Ltd [http://www.medjaden.com/index.html] for providing English language editing services.

\section{References}

[1] N.H. Shoukry, A.G. Cawthon, C.M. Walker, Cell-mediated immunity and the outcome of hepatitis C virus infection, Annu. Rev. Microbiol. 58 (2004) 391-424.

[2] M.J. Koziel, Cellular immune responses against hepatitis C virus, Clin. Infect. Dis. 41 (Suppl. 1) (2005) S25-S31.

[3] C.T. Tseng, G.R. Klimpel, Binding of the hepatitis C virus envelope protein E2 to CD81 inhibits natural killer cell functions, J. Exp. Med. 195 (2002) 43-49.

[4] K. Melen, R. Fagerlund, M. Nyqvist, P. Keskinen, I. Julkunen, Expression of hepatitis C virus core protein inhibits interferon-induced nuclear import of STATs, J. Med. Virol. 73 (2004) 536-547.

[5] K. Li, E. Foy, J.C. Ferreon, M. Nakamura, A.C. Ferreon, M. Ikeda, et al., Immune evasion by hepatitis C virus NS3/4 A protease-mediated cleavage of the Toll like receptor 3 adaptor protein TRIF, Proc. Natl. Acad. Sci. U. S. A. 102 (2005) 2992-2997.

[6] S. Khaliq, S. Jahan, A. Pervaiz, Sequence variability of HCV Core region: important predictors of HCV induced pathogenesis and viral production, Infect. Genet. Evol. 11 (2011) 543-556.

[7] J. Dubuisson, Hepatitis C virus proteins, World J. Gastroenterol. 13 (2007) 2406-2415.

[8] S. Jahan, U.A. Ashfaq, S. Khaliq, B. Samreen, N. Afzal, Dual behavior of HCV Core gene in regulation of apoptosis is important in progression of HCC, Infect. Genet. Evol. 12 (2012) 236-239.

[9] M.H. Heim, D. Moradpour, H.E. Blum, Expression of hepatitis C virus proteins inhibits signal transduction through the Jak-STAT pathway, J. Virol. 73 (1999) 8469-8475.

[10] A. Basu, K. Meyer, R.B. Ray, R. Ray, Hepatitis C virus core protein modulates the interferon-induced transacting factors of Jak/Stat signaling pathway but does not affect the activation of downstream IRF-1 or 561 gene, Virology 288 (2001) 379-390.

[11] E. Luquin, E. Larrea, M.P. Civeira, J. Prieto, R. Aldabe, HCV structural proteins interfere with interferon-alpha Jak/STAT signalling pathway, Antivir. Res. 76 (2007) 194-197.

[12] C. Auffray, M.H. Sieweke, F. Geissmann, Blood monocytes: development, heterogeneity, and relationship with dendritic cells, Annu. Rev. Immunol. 27 (2009) 669-692.

[13] N. Kadowaki, S. Ho, S. Antonenko, R.W. Malefyt, R.A. Kastelein, F. Bazan, et al., Subsets of human dendritic cell precursors express different Toll-like receptors and respond to different microbial antigens, J. Exp. Med. 194 (2001) 863-869.

[14] I. Bekeredjian-Ding, S.I. Roth, S. Gilles, T. Giese, A. Ablasser, V. Hornung, et al., T cellindependent, TLR-induced IL-12p70 production in primary human monocytes, J. Immunol. 176 (2006) 7438-7446.

[15] E. Niesen, J. Schmidt, T. Flecken, R. Thimme, Suppressive effect of interleukin 10 on priming of naive hepatitis C virus-specific CD8 ${ }^{+}$T cells, J. Infect. Dis. 211 (2015) 821-826.

[16] B.S. Liu, Z.M. Groothuismink, H.L. Janssen, A. Boonstra, Role for IL-10 in inducing functional impairment of monocytes upon TLR4 ligation in patients with chronic HCV infections, J. Leukoc. Biol. 89 (2011) 981-988.

[17] J. Jabłońska, T. Pawłowski, T. Laskus, M. Zalewska, M. Inglot, S. Osowska, et al., The correlation between pretreatment cytokine expression patterns in peripheral blood mononuclear cells with chronic hepatitis c outcome, BMC Infect. Dis. 15 (2015) 556.

[18] S.D. Blackburn, E.J. Wherry, IL-10, T cell exhaustion and viral persistence, Trends Microbiol. 15 (2007) 143-146.

[19] E.Y. Chang, B. Guo, S.E. Doyle, G. Cheng, Cutting edge: involvement of the type I IFN production and signaling pathway in lipopolysaccharide-induced IL-10 production, J. Immunol. 178 (2007) 6705-6709.

[20] Y.T. Tsai, S.Y. Chang, C.N. Lee, C.L. Kao, Human TLR3 recognizes dengue virus and modulates viral replication in vitro, Cell. Microbiol. 11 (2009) 604-615. 
[21] A.L. Zignego, D. Macchia, M. Monti, V. Thiers, M. Mazzetti, M. Foschi, et al., Infection of peripheral mononuclear blood cells by hepatitis C virus, J. Hepatol. 15 (1992) 382-386.

[22] X.L. Pang, H.X. Song, Q.Q. Zhang, Z.K. Tu, J.Q. Niu, Hepatitis C virus regulates the production of monocytic myeloid-derived suppressor cells from peripheral blood mononuclear cells through PI3K pathway and autocrine signaling, Clin. Immunol. 164 (2016) 57-64.

[23] Y.P. Ho, I.S. Sheen, C.T. Chiu, C.S. Wu, C.Y. Lin, A strong association between downregulation of HLA-DR expression and the late mortality in patients with severe acute pancreatitis, Am. J. Gastroenterol. 101 (2006) 1117-1124.

[24] A. Chéron, G. Monneret, C. Landelle, B. Floccard, B. Allaouchiche, Low monocytic HLA-DR expression and risk of secondary infection, Ann. Fr. Anesth. Reanim. 29 (2010) 368-376.

[25] M.E. Cramp, P. Carucci, J. Underhill, N.V. Naoumov, R. Williams, P.T. Donaldson, Association between HLA class II genotype and spontaneous clearance of hepatitis C viraemia, J. Hepatol. 29 (1998) 207-213.

[26] A. Mangia, R. Gentile, I. Cascavilla, M. Margaglione, M.R. Villani, F. Stella, et al., HLA class II favors clearance of HCV infection and progression of the chronic liver damage, J. Hepatol. 30 (1999) 984-989.

[27] E.J. Minton, D. Smillie, K.R. Neal, W.L. Irving, J.C. Underwood, V. James, Association between MHC class II alleles and clearance of circulating hepatitis C virus, J. Infect. Dis. 178 (1998) 39-44.

[28] I. Zubkova, H. Duan, F. Wells, H. Mostowski, E. Chang, K. Pirollo, et al., Hepatitis C virus clearance correlates with HLA-DR expression on proliferating CD8 ${ }^{+}$T cells in immune-primed chimpanzees, Hepatology 59 (2014) 803-813.

[29] A. Perrella, P. Conca, G. Perrella, Tarantino, $\mathrm{CD}^{+} / \mathrm{CD}^{2} 8^{+}$: immune activity and clinical significance in HCV patients with and without interferon therapy, Eur. Rev. Med. Pharmacol. Sci. 4 (2000) 127-131.

[30] J.K. Flynn, G.J. Dore, M. Hellard, B. Yeung, W.D. Rawlinson, P.A. White, et al., Maintenance of Th1 HCV-specific responses in individuals with acute HCV who achieve sustained virological clearance after treatment, J. Gastroenterol. Hepatol. 28 (2013) 1770-1781.

[31] J. Zheng, H. Liang, C. Xu, Q. Xu, T. Zhang, T. Shen, et al., An unbalanced PD-L1/CD86 ratio in $\mathrm{CD} 14(++) \mathrm{CD} 16(+)$ monocytes is correlated with HCV viremia during chronic HCV infection, Cell. Mol. Immunol. 11 (2014) 294-304.

[32] T. Shen, X. Chen, Y. Chen, O Xu, F. Lu, S. Liu, Increased PD-L1 expression and PD-L1/ CD86 ratio on dendritic cells were associated with impaired dendritic cells function in HCV infection, J. Med. Virol. 82 (2010) 1152-1159.
[33] Y.W. Liu, H.P. Tseng, L.C. Chen, B.K. Chen, W.C. Chang, Functional cooperation of simian virus 40 promoter factor 1 and CCAAT/enhancer-binding protein beta and delta in lipopolysaccharide-induced gene activation of IL-10 in mouse macrophages, J. Immunol. 171 (2003) 821-828.

[34] S. Cao, X. Zhang, J.P. Edwards, D.M. Mosser, NF-kappaB1 (p50) homodimers differentially regulate pro- and anti-inflammatory cytokines in macrophages, J. Biol. Chem. 281 (2006) 26041-26050.

[35] S. Cao, J. Liu, L. Song, X. Ma, The protooncogene c-Maf is an essential transcription factor for IL-10 gene expression in macrophages, J. Immunol. 174 (2005) 3484-3492.

[36] H.D. Brightbill, S.E. Plevy, R.L. Modlin, S.T. Smale, A prominent role for Sp1 during lipopolysaccharide-mediated induction of the IL-10 promoter in macrophages, J. Immunol. 164 (2000) 1940-1951.

[37] A. Dolganiuc, S. Oak, K. Kodys, D.T. Golenbock, R.W. Finberg, E. Kurt-Jones, et al. Hepatitis C core and nonstructural 3 proteins trigger toll-like receptor 2-mediated pathways and inflammatory activation, Gastroenterology 127 (2004) 1513-1524.

[38] U. Düesberg, A. von dem Bussche, C. Kirschning, K. Miyake, T. Sauerbruch, U. Spengler, Cell activation by synthetic lipopeptides of the hepatitis $\mathrm{C}$ virus (HCV)-core protein is mediated by toll like receptors (TLRs) 2 and 4, Immunol. Lett. 84 (2002) 89-95.

[39] K. Kato, E.P. Lillehoj, K.C. Kim, MUC1 regulates epithelial inflammation and apoptosis by polyl: $C$ through inhibition of toll/IL-1 receptor-domain-containing adapter-inducing IFN- $\beta$ (TRIF) recruitment to toll-like receptor 3, Am. J. Respir. Cell Mol. Biol. 51 (2014) 446-454.

[40] Z. Huang, C. Gao, X. Chi, Y.W. Hu, L. Zheng, T. Zeng, et al., IL-37 expression is upregulated in patients with tuberculosis and induces macrophages towards an M2-like phenotype, Scand. J. Immunol. 82 (2015) 370-379.

[41] A. Guilmot, J. Bosse, Y. Carlier, C. Truyens, Monocytes play an IL-12-dependent crucial role in driving cord blood NK cells to produce IFN-g in response to Trypanosomacruzi, PLoS Negl. Trop. Dis. 7 (2013) e2291.

[42] B. Xiu, Y. Lin, D.M. Grote, S.C. Ziesmer, M.P. Gustafson, M.L. Maas, et al., IL-10 induces the development of immunosuppressive CD14(+)HLA-DR(low/-) monocytes in Bcell non-Hodgkin lymphoma, blood, Cancer J. 5 (2015), e328.

[43] S. Ito, P. Ansari, M. Sakatsume, H. Dickensheets, N. Vazquez, R.P. Donnelly, et al., Interleukin-10 inhibits expression of both interferon alpha- and interferon gamma-induced genes by suppressing tyrosine phosphorylation of STAT1, Blood 93 (1999) 1456-1463. 